\title{
Seeking physical/mathematical coherence by recruiting and reconciling reasoning: A case study in E\&M
}

\author{
Gabriel S. Ehrlich \\ Departments of Physics and Curriculum \& Instruction, University of Illinois Urbana-Champaign, 1110 W Green St, Urbana, IL, 61801 \\ Katherine Gifford \\ Department of Physics, Adelphi University, 1 South Ave, Garden City, NY, 11530 \\ Eric Kuo \\ Departments of Physics and Curriculum \& Instruction, University of Illinois Urbana-Champaign, 1110 W Green St, Urbana, IL, 61801 \\ Engin Bumbacher \\ Haute École Pédagogique Vaud, Avenue de Cour 33, 1014 Lausanne, Switzerland
}

\begin{abstract}
The entanglement between physical and mathematical reasoning can be exploited for problem solving, making physical/mathematical coherence seeking a desirable learning goal. Our research aim is to contribute an empirical example of physical/mathematical coherence seeking during physics problem solving. Drawing from a video corpus of 18 interviews with students solving qualitative E\&M problems, we present a case study of how and why a student reasons coherently with physical entities/processes and mathematical equations across three lines of reasoning. We focus especially on the role of the student's attempt to seek coherence, which explains their serious consideration of a contradiction that arises between a physical and a mathematical line of reasoning. Even after reaching the correct conclusion, the student recruits a third line of reasoning involving ideas from outside the explicit problem statement to resolve the contradiction. We end by discussing hypotheses about how instruction in physical/mathematical coherence seeking could support students in problem solving.
\end{abstract}

\footnotetext{
2021 PERC Proceedings edited by Bennett, Frank, and Vieyra; Peer-reviewed, doi.org/10.1119/perc.2021.pr.Ehrlich

Published by the American Association of Physics Teachers under a Creative Commons Attribution 4.0 license.

Further distribution must maintain the cover page and attribution to the article's authors.
} 


\section{INTRODUCTION}

The physical and mathematical facets of physics knowledge are inseparable [1]. Physicists' equation use, for example, reflects inextricable physical meaning [2]. Just as researchers exploit this relationship to make discoveries, some students access this "blended" picture during problem solving and use it to identify shortcuts and make physical sense of equations [3]. In models of physics problem solving, processes connecting the physical and mathematical include modeling, mathematization, and interpretation [1-2].

The problem-solving advantages of reasoning at the interface of physics and mathematics make it a desirable learning goal. Aligned with calls to help students learning science seek coherence (alignments of disparate scientific ideas) [4], we ask how students might seek coherence in physics problem solving - specifically coherence between physical and mathematical reasoning. For example, physics students might check answers with dimensional/unit analysis, limiting cases, or reasonable values $[5,6]$. These solution checking techniques interpret symbolic mathematical answers to physics problems as having a coherent physical meaning.

Other coherent reasoning techniques could be used during any stage of problem solving. Reasoning elements that might be used in seeking coherence include purely qualitative treatments of physical entities, numerical values, pprims [7], units, proportional reasoning, limiting cases, symbolic forms [8], and equations given physical meaning. Analogical reasoning between related systems can take place at various levels of mathematization [1]. Within proportional reasoning are various gradations of mathematization, from more/less/equal (no numerical proportion) to equations that include the constant of proportionality [1]. And excursions to formal calculation can return results that are unfinished until they are imbued with physical meaning [2].

Our knowledge of coherent reasoning in physics lacks a systematic understanding of its dynamics in students' problem solving. In the video case study we present in this paper, we study how and why a student uses coherent physi$\mathrm{cal} /$ mathematical reasoning when answering a qualitative physics problem. We focus especially on the role of the student's attempt to seek coherence, which drives their problem-solving decisions. We discuss the value of teaching students to seek physical/mathematical coherence and some ideas for integrating it into instruction.

\section{METHODS}

The data for this paper come from a corpus of video-recorded interviews designed and conducted by the fourth author. The interview protocol consisted of qualitative E\&M problems on the topics of electric charge, electric fields, simple circuits, and capacitors. Students were recruited from two institutions of higher education on the U.S. West Coast: 9 students taking physics at a community college, 6 undergraduate physics or engineering students at an elite private research university, and 3 physics or engineering graduate students at the same private university. Each interview lasted about one hour. The interviewer presented the problems and asked follow-up questions to clarify students' thinking.

In the first stage of analysis, one researcher watched each video-recorded interview while writing a content log, which summarized the conversation and reasoning at roughly 30 to 90 -second intervals, as needed to capture the timestamp accurately. Excerpts that caught the researchers' attention were brought to weekly meetings of the first three authors to share, where we would watch the excerpt, pose questions about the student's reasoning, and progressively refine our hypotheses in response to new observations [9]. We attended to students' frequent switching between equation use and discussion of physical entities and processes within the circuit. Across multiple interviews, two focal problems from the protocol emerged as eliciting especially rich examples of reasoning that combined equations and physical concepts.

In the second stage of analysis, we zoomed in on several episodes of student problem solving on one of these focal problems, Four Circuits. Each episode of interest was initially transcribed verbatim. Then it was annotated as necessary over the course of analysis to capture relevant details, including paraverbal cues, pauses, writing, facial expressions, and gestures. The transcript and video were interpreted by the first author to construct arguments about students' reasoning, which they would bring to meetings for discussion and revision. This paper discusses a single case of one student working on Four Circuits, chosen for the student's overt references to reasoning and coherence and their detailed descriptions of their thinking.

"Blake" is a private university undergraduate student entering their second year of study. We read Blake as a white man, which we acknowledge may have influenced our analytic interpretations. Because we did not ask Blake's pronouns, we refrain from assumption and instead refer to Blake using they/them pronouns throughout.

\section{A. The Four Circuits focal problem}

In the Four Circuits focal problem, students are given diagrams of four circuits, depicted in Fig. 1. The capacitors in circuits $\mathrm{A}, \mathrm{B}$, and $\mathrm{C}$ have the same plate separation distance, and in circuit D the separation distance is half as much. All capacitors are initially fully charged at the same voltage. Students are asked to determine which capacitors have the most and least charge remaining after the capacitors have been allowed to discharge for the same amount of time.

In terms of a capacitor's area $A$ and distance $d$, the capacitance is $C=A /\left(\epsilon_{0} d\right)$, with $\epsilon_{0}$ a constant (the permittivity of free space). Because $d$ is in the denominator, a capacitor with a smaller separation distance will have a larger capacitance (assuming the area is the same). This gives the capacitor in circuit $\mathrm{D}$ a larger initial charge, since $Q=C V$. 

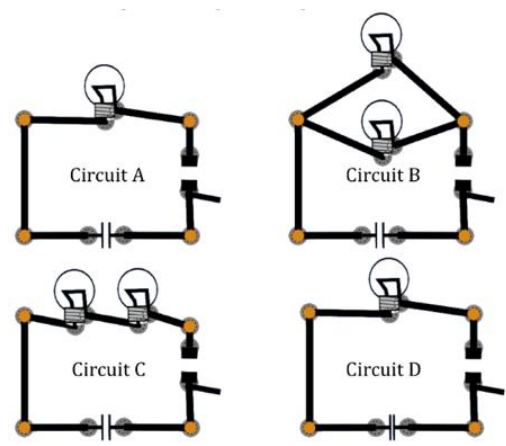

FIG. 1. The circuit diagrams in the Four Circuits problem.

Circuit $\mathrm{B}$ has the smallest time constant $R C$ and therefore the least charge remaining. Circuit $\mathrm{D}$ has the most, because it has the same time constant as circuit $\mathrm{C}$ and starts with more charge.

\section{RESULTS}

\section{A. Recruiting three lines of reasoning}

\section{The first line of reasoning: Electrons Jumping}

As the interviewer (I) reads the part of the prompt explaining that the capacitor in circuit $\mathrm{D}$ has half the distance between its plates compared to the other capacitors, Blake (B) starts reasoning about how the separation distance affects the capacitance.

B: Okay. But the capacitance is different.

I: How is it different?

B: Um. How is, I mean it's, the configuration of capacitors, having the, the distance of the plates is an important factor in, um, in the capacitance.

I: So then is the capacitance bigger, smaller, or the same. As (points to A, B, C).

B: Right. Um... (9 s pause) Well, I can't remember the equation. But, thinking, logically, um, my guess is that having the plates closer, it's more likely that electrons can jump across, kind of, maybe? (furrows brow and turns head sideways) And therefore the capacitance would be smaller.

Here, Blake indicates two ways of reasoning about the relationship between capacitance and distance. One is through an equation, which they say they cannot remember. The other is through the physical mechanism of electrons jumping across the gap, which they use for proportional reasoning when they conclude that less separation means less capacitance. We call this line of reasoning Electrons Jumping. Although the mechanistic explanation of capacitance and the conclusion are canonically incorrect, this excerpt shows how an obstacle in one line of reasoning can be circumvented by using another line of reasoning. Here, a mathematical line of reasoning is obstructed, and a physical line of reasoning is the detour.

\section{The second line of reasoning: Capacitor Equations}

After the interviewer finishes explaining the question prompt, Blake starts ranking the circuits when they run into an issue that leads them to change their reasoning.

B: Okay. So I'm starting out by saying this [D] has already less (writes "less" underneath D). (unintelligible) capacitance the least. But it's going to discharge at different rates based on the resistance, um. So the resistance here $[\mathrm{C}]$ is the greatest. Because it's two in series. So, yeah, these $[A$ and $D]$ are the same, um, and this $[\mathrm{B}]$ is the least, um. So this [B]...but now you have this [D], like, (laughing) because it [D] has less charge to begin with, but then it [B] has, discharges faster, um.

I: A lot going on?

B: Well, it's like these two opposing variables kind of thing, that like, um, which, leads me to believe if this conclusion ["less" written under D] is correct at the beginning, then I wouldn't be able to distinguish, just kind of, reasoning it out, which one has more charge. So therefore, maybe this [D] should be a greater capacitance, because that might allow me to, um... (7 s pause) $\mathrm{C}$ equals $\mathrm{Q}$ over V? (writes " $C=Q / V$ ”) I think that's the right... Having the plates closer together, um... (7 s pause) V equals $E \mathrm{D}$. (under previous equation, writes " $V=e d$ " [sic]) Equals Q over E D. (after " $C=Q / V$," writes " $=Q / E d$ ') So having... lower... so maybe this (points to " $C=Q / V=Q / E d$ ") is more accurate. Having a... (leans head on hand, elbow on table) smaller, um... (taps pencil on paper three times) distance increases the capacitance.

Blake starts this excerpt by correctly ranking the total resistances of the circuit but realizes that there are two opposing variables when comparing circuits B and D: B has a smaller resistance but D has a smaller initial charge (consistent with the previous reasoning that $\mathrm{D}$ would have a smaller capacitance). Given these assumptions, Blake notes that these two opposing variables make it impossible to qualitatively reason out which one has more charge using their current line of reasoning. The idea of two opposing influences has been identified previously as a "conceptual schema" [8], which physics students can use to conclude incorrectly that these two opposing influences exactly compensate for one another [11-15]. However, here Blake explicitly notes that a definite determination cannot be made. Seemingly because their current line of reasoning cannot qualitatively rank circuits $\mathrm{B}$ and $\mathrm{D}$, they reconsider the capacitance of circuit $\mathrm{D}$.

Specifically, Blake considers whether D has a greater capacitance, in contrast to the conclusion from Electrons Jumping. Their speech trails off, and they proceed to derive an equation relating $C$ to $d$, writing down $C=Q / V=Q / E d$. Blake concludes from their derived equation that a smaller distance between capacitor plates increases the capacitance, 
which would follow from the inversely proportional dependence of $C$ on $d$, though this is not explicitly explained by Blake. This is the correct dependence, and the reasoning is consistent with an isolated capacitor. We call this mathematical line of reasoning, which relies on algebraic manipulation and proportional reasoning with equations, Capacitor Equations. By forecasting that the conclusion of Electrons Jumping will present an opposing variables problem and undertaking Capacitor Equations instead, Blake again circumvents an obstacle in one line of reasoning by switching to another. Here, Blake switches from physical to mathematical reasoning. This can even be interpreted as a switch back to mathematical reasoning, since Blake begins by noting a failed attempt to recall the relevant equation relating capacitance and distance. Blake is able to rederive an equation with the relevant relationship rather than recall the original.

Blake does compare Capacitor Equations favorably against Electrons Jumping, calling it "more accurate." However, the hedges "maybe" and "I think," their long pauses, and their body language do not convey confidence in the result. Possibly because of ongoing doubt about the correct form of the forgotten equation, they do not immediately proceed with their revised assumption.

\section{The third line of reasoning: Dielectric Analogy}

After Blake uses the equation to conclude that the smaller plate separation distance increases the capacitance, the interviewer apparently interrupts Blake's thoughts with the question, "Why would that be?" The interviewer's second sentence is interrupted by Blake's overlapping speech. (Overlapping speech is enclosed by curly brackets. The double A in "whaat" denotes a lengthened vowel.)

I: Why would that be? (10 s pause) Just trying to, \{make sense of your reasoning .

B: \{Right, right, right $\}$, exactly. Um, and now I am trying to piece, like, because I do remember something about the ability for electrons to jump over, I mean that was one of the reasons, I mean, also piecing in dielectrics, the reason for improving a dielectric, adding a dielectric increases the capacitance. And what does a dielectric do? It decreases the, uh, the voltage difference between there. Um, and so having the plates closer together... wait, whaat? Having the plates closer together... increases the voltage difference. No wait, we just said the voltage was, um... electric field times distance... (4 s pause) Sorry, yeah yeah, having the plates closer together decreases the potential difference, if we're assuming constant electric field, wait, so yeah, so there's a, a lower electric, smaller electric field...

One could interpret the interviewer's question, "Why would that be?", as trying to understand Blake's equationbased reasoning in the previous excerpt. However, Blake's response concerns physical models, showing that they are framing the question as requesting a physical explanation that coheres with their equation-based conclusion. In their response, they bring up two physical ideas: Electrons Jumping, and the scenario of adding a dielectric compared to decreasing the plate separation distance.

There are indications that Blake has recognized and is trying to resolve the contradiction between the conclusions of Electrons Jumping and Capacitor Equations. They mention "trying to piece" and "piecing in," which may suggest the metaphor of solving a jigsaw puzzle-here, trying to get the different pieces of reasoning to fit together-and the recognition that the pieces are not currently fitting. In addition, Blake's comment "because I do remember [...]" regarding the idea behind Electrons Jumping uses the emphasizing word "do," which may convey reinforcing the idea against a reason to doubt it. We interpret this emphasis as Blake's recognition of the contradiction between the remembered mechanism of Electrons Jumping and their current reasoning with Capacitor Equations.

This recognition of the contradiction potentially explains why Blake would introduce dielectrics at all. Although the problem does not mention dielectrics, Blake introduces this idea and follows it up by seeing if adding a dielectric and reducing capacitor plate separation affect the voltage difference in the same way - a new, third line of reasoning we call Dielectric Analogy. The introduction of this physical line of reasoning makes sense if Blake recognizes the contradiction between Electrons Jumping and Capacitor Equations and is seeking a new physical explanation that is consistent with the mathematics of Capacitor Equations. Because the dielectric increases capacitance and decreases voltage, finding that decreasing plate separation decreases voltage as well could provide a physical mechanism explaining the mathematical result that capacitance would increase. In other words, Dielectric Analogy is a physical mechanism coherent with the mathematical reasoning in Capacitor Equations.

Blake also leverages coherence between physical and mathematical reasoning within Dielectric Analogy itself by incorporating the equation $V=E d$. Because $V$ is directly proportional to $d$, decreasing the plate separation would decrease the voltage difference. This could be the tacit reasoning behind Blake's correction ("Sorry, yeah yeah") which is needed to complete the physical analogy with adding a dielectric. This demonstrates how episodes of seeking coherence in student thinking can be more complex than one simple line of physical reasoning cohering with one simple line of mathematical reasoning.

Several moments in this excerpt support an interpretation that Blake expects coherence between Capacitor Equations and their physical reasoning. First, Blake frames the interviewer's question as requesting a physical explanation of their mathematical result. Then, they recognize the contradiction and attempt to resolve it by explaining Capacitor Equations with a novel physical analogy. Finally, they draw on an equation to support their physical reasoning. Blake seeks coherence more overtly in the next excerpt. 


\section{B. Reconciling lines of reasoning}

In this excerpt, Blake sums up what they conclude from these three lines of reasoning. Following Dielectric Analogy and after a brief digression where Blake discusses the difficulty of the problem with the interviewer, they continue:

B: So let me, for now, say that, perhaps, yeah, my first reasoning was a little, wrong, and that, actually having the plates closer together, um, decreases the voltage difference, which increases the capacitance. I think that also agrees with, the equation $[C=Q / V=Q / E d]$ that maybe, is right? I don't remember, um... And then if that case, then this [D] has the largest charge... Um, to begin with...

In the first sentence, Blake refers to their first reasoning (likely Electrons Jumping) being incorrect and concludes that decreasing the plate separation increases the capacitance (which disagrees with Electrons Jumping). There is evidence that Blake is seeing this conclusion as coming from Dielectric Analogy, since they mention the decrease in voltage - an idea only present in Dielectric Analogy - and they say that this "also agrees with" the equation from the remaining line of reasoning, Capacitor Equations.

This excerpt shows Blake reconciling their three lines of reasoning. It appears that coherence between Dielectric Analogy and Capacitor Equations helps Blake be more certain that decreasing plate separation will increase capacitance, and it helps them reject the conclusion of Electrons Jumping despite remembering the physical mechanism of electrons jumping from one capacitor plate to another.

In getting to this point, Blake recruited and reconciled three lines of reasoning. It was not logically necessary for them to seek a physical explanation for Dielectric Equations; in the second excerpt, they already evaluated it as "more accurate" than Electrons Jumping. Nor was it necessary to reconcile the three lines of reasoning once constructed; in this excerpt, they could rely only on Dielectric Analogy when they say "if that case [sic]" and proceed to determine the relative charge on the capacitor in circuit $\mathrm{D}$. Why did they engage in multiple lines of reasoning and seek to reconcile them? We propose that Blake's decisions reflect a valuing of coherence between physical and mathematical reasoning. If two lines of reasoning are correct, they should agree, yet the conclusions of Electrons Jumping and Capacitor Equations appeared contradictory. Here, Blake finds some resolution, discarding one line of physical reasoning (Electrons Jumping) in light of another (Dielectric Analogy). The new physical picture seems to agree with Capacitor Equations, offering coherence with Blake's mathematical reasoning.

Coherence between physical and mathematical reasoning may also provide support for a conclusion if there is uncertainty about individual lines of reasoning. Blake indicates uncertainty about the derived equations in Capacitor Equations by saying "[it] maybe, is right? I don't remember," which echoes when they noted initially that they "can't remember the equation" relating separation distance to capacitance. New reasoning (here, the physical reasoning of Dielectric Analogy) may through its coherence with another (here, Capacitor Equations) buffer it against uncertainty and help the student trust the common conclusion.

\section{DISCUSSION}

We have described the dynamics of Blake's reasoning in this episode as driven by seeking coherence [4] between their physical and mathematical reasoning. Seeking coherence explains Blake's serious consideration of the contradictions that arose between two of their lines of reasoning. It also explains why Blake brings in ideas from outside the explicit problem statement to reason about the problem even after reaching the correct conclusion. Blake's successful workaround for a forgotten equation and their decision to discard an incorrect physical model showcase the value of seeking coherence during problem solving.

This case provides a hypothesis for why explicit instruction in solution checks can produce script-like student implementation of solution checks rather than more fluid and rich checking behaviors [5]. Here, we propose that the richness of how Blake checks their answer is driven by coherence seeking, not by solution-checking techniques alone. Perhaps instruction that focuses on the underlying aim of seeking coherence (in conjunction with instruction on explicit reasoning strategies) may foster more fluid and less scripted solution-checking behavior.

In addition to explicitly seeking coherence, they exploit physical/mathematical coherence through a variety of reasoning elements at different levels of mathematization: proportional reasoning with a physical mechanism, proportional reasoning with an equation, a physical analogy, and an equation incorporated into physical reasoning. These and other elements may play an everyday role in expert physics problem solving. Taking inventory of these structural (mathematical) skills [1] could offer specific instructional targets to support physical/mathematical coherence seeking.

\section{ACKNOWLEDGMENTS}

This material is based upon work supported by the National Science Foundation and Department of Defense under Grants PHY-1659598 and PHY-1950744. Any opinions, findings, and conclusions or recommendations expressed in this material are those of the author(s) and do not necessarily reflect the views of the National Science Foundation or Department of Defense. 
[1] O. Uhden, R. Karam, M. Pietrocola, and G. Pospiech, Modelling Mathematical Reasoning in Physics Education, Sci \& Educ 21, 485 (2012).

[2] E. F. Redish and E. Kuo, Language of Physics, Language of Math: Disciplinary Culture and Dynamic Epistemology, Sci \& Educ 24, 561 (2015).

[3] E. Kuo, M. M. Hull, A. Gupta, and A. Elby, How Students Blend Conceptual and Formal Mathematical Reasoning in Solving Physics Problems, Science Education 97, 32 (2013).

[4] T.-R. Sikorski and D. Hammer, Looking for Coherence in Science Curriculum, Science Education 101, 929 (2017).

[5] T.-R. Sikorski, G. D. White, and J. Landay, Uptake of Solution Checks by Undergraduate Physics Students, in PERC Proceedings (2018), pp. 368-371.

[6] K. T. Hahn, P. J. Emigh, M. Lenz, and E. Gire, Student SenseMaking on Homework in a Sophomore Mechanics Course, in PERC Proceedings (2018), pp. 160-163.

[7] A. A. diSessa, Toward an Epistemology of Physics, Cognition and Instruction 10, 105 (1993).

[8] B. L. Sherin, How Students Understand Physics Equations, Cognition and Instruction 19, 479 (2001).

[9] R. A. Engle, F. R. Conant, and J. G. Greeno, Progressive Refinement of Hypotheses in Video-Supported Research, in
Video Research in the Learning Sciences, edited by R. Goldman, R. Pea, B. Barron, and S. J. Derry (2007), pp. 239-254.

[10] D. Tannen, editor, Framing in Discourse (Oxford University Press, 1993).

[11] M. Kryjevskaia, M. R. Stetzer, and N. Grosz, Answer first: Applying the heuristic-analytic theory of reasoning to examine student intuitive thinking in the context of physics, Phys. Rev. ST Phys. Educ. Res. 10, 020109 (2014).

[12] R. A. Lawson and L. C. McDermott, Student understanding of the work-energy and impulse-momentum theorems, Am. J. Phys. 55, 811 (1987).

[13] B.W. Frank, S. E. Kanim, and L. S. Gomez, Accounting for variability in student responses to motion questions, Phys. Rev. ST Phys. Educ. Res. 4, 020102 (2008).

[14] M. E. Loverude, C. H. Kautz, and P. R. Heron, Helping students develop an understanding of Archimedes' principle. I. Research on student understanding, Am. J. Phys. 71, 1178 (2003).

[15] E. Kuo, M. M. Hull, A. Elby, and A. Gupta, Assessing Mathematical Sensemaking in Physics through Calculation-Concept Crossover, Phys. Rev. Phys. Educ. Res. 16, 020109 (2020). 